\title{
Editorial
}

\section{CANTIDAD O CALIDAD DE LAS PUBLICACIONES EN ODONTOLOGÍA: "SER O NO SER"}

El incremento de la productividad de la literatura científica en odontología ha ido en aumento de manera significativa en las últimas décadas, debido a los avances científicos y tecnológicos que promueven la generación de nuevos conocimientos y el análisis de la información existente. El tener acceso a esta información hoy en día es más fácil, debido a diversidad de las fuentes de información odontológica que se encuentran disponibles a través de bases de datos y sitios de acceso abierto denominados "Open Access" como: Pubmed, Elsevier, Scopus, Researchgate, DOAJ, Scielo, Google Académico, LaReferencia.info, entre otros. Esto nos lleva a realizarnos las siguientes preguntas respecto a la información; ¿Dónde busco?, ¿Qué revistas o Journals seleccionó?,¿Cuántos artículos analizo?, ¿Cuáles tienen calidad y cuáles no?, todas estas preguntas tienen que ver con el tema de cantidad o calidad; la gran pregunta es: ¿Cómo y quién evalúa la información?.

Actualmente existen indicadores bibliométricos que determinan la calidad de las publicaciones. Desde el enfoque cuantitativo existen indicadores que evalúan el número de veces que un artículo ha sido citado como es el factor de Impacto del Journal of Citation Reports (JCR), el Índice SJR (SCImagoJournal Rank), Índice H, Índice i10 que permiten comparar distintos Journals, Revistas. Sin embargo hablando en términos de calidad, estos índices no permiten evaluar el contenido del artículo, para poder hacer un análisis de la literatura científica se deben utilizar criterios metodológicos que se encuentran disponibles en instrumentos de evaluación de la evidencia como el modelo inglés: Oxford Center for Evidence-Based Medicine (OCEBM), que evalúa y jerarquiza la evidencia dependiendo del área clínica, tipo de estudio, diagnóstico, diagnóstico diferencial, etiología, análisis de la frecuencia epidemiológica y decisiones clínicas.Otro instrumento de evaluación es él: Grading of Recommendations, Assessment, Development and Evaluation (GRADE), que evalúa la calidad de la evidencia científica de: Guías de Práctica Clínica, revisiones sistemáticas, clasificando la evidencia de: (muy baja, baja, moderada y alta) de acuerdo a valores y preferencias que los pacientes reportan en estudios clínicos publicados.

Es importante resaltar que existe una diversidad de instrumentos para evaluar la calidad de la evidencia científica de los datos epidemiológicos, clínicos y experimentales jerarquizando y evaluando la calidad de los resultados publicados con el propósito de tomar mejores decisiones 
clínicas y diseñar estudios clínicos, epidemiológicos y experimentales. Por lo que es necesario utilizar al menos un instrumento para evaluar de manera sistematizada.

Od. Esp Daniela Carmona Ruiz. Editora de la Revista Odontológica Mexicana Facultad de Odontología, UNAM.

Dr. César Esquivel Chirino Profesor del Área de Básicas Médicas e Investigación Clínica de la División de Estudios de la Facultad de Odontología de la UNAM. 